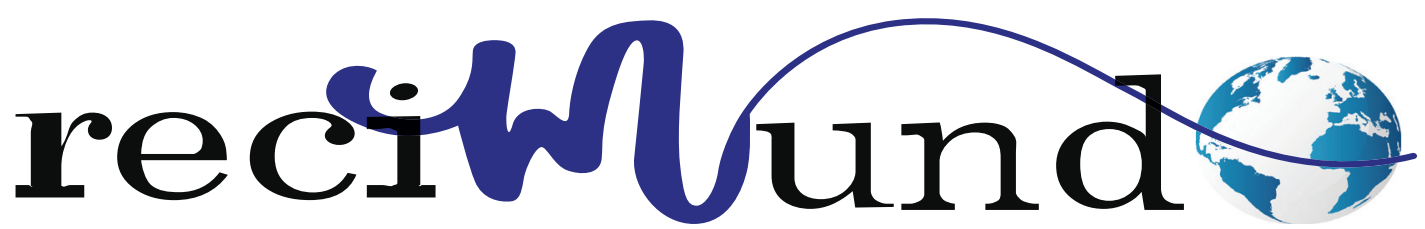

Revista Científica Mundo de la Investigación y el Conocimiento

DOI: $10.26820 /$ recimundo/5.(1).enero.2021.313-321

URL: http://recimundo.com/index.php/es/article/view/1014

EDITORIAL: Saberes del Conocimiento

REVISTA: RECIMUNDO

ISSN: 2588-073X

TIPO DE INVESTIGACIÓN: Artículo de Revisión

Código UNESCO: 32 Ciencias Médicas

PAGINAS: $313-321$

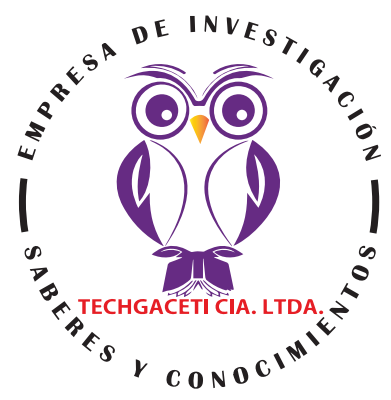

\title{
Síntomas y tratamiento al neonato con hipoacusia
}

Symptoms and treatment of the neonate with hearing loss

Sintomas e tratamento do neonatal com perda auditiva

Andrea Natacha Pico Tagle1; Julio Josué Rodríguez Centeno2; Roger Adalberto Borja Zambrano3;

Diana Aracely Mero Varela ${ }^{4}$

\section{RECIBIDO: 28/11/2020 ACEPTADO: 06/12/2020 PUBLICADO: 31/01/2021}

1. Médica Cirujana; Médico General en Funciones Hospitalarias; Hospital Instituto Ecuatoriano de Seguridad Social; Manta, Ecuador; natachapicotagle@hotmail.com; https://orcid.org/0000-0003-4370-3630

2. Médico Cirujano; Médico General en Funciones Hospitalarias; Hospital Instituto Ecuatoriano de Seguridad Social; Manta, Ecuador; julio_24jj@hotmail.com; https://orcid.org/0000-0003-4595-111X

3. Médico Cirujano; Médico General en Funciones Hospitalarias Hospital Instituto Ecuatoriano de Seguridad Social; Médico Residente de Unidad de Cuidados Intensivos en Clínica del Sol; Manta, Ecuador; roger932009@hotmail.com; https://orcid. org/0000-0002-5267-8257

4. Médico General; Médico General en Funciones Hospitalarias; Hospital Instituto Ecuatoriano de Seguridad Social; Médico Residente Clínica Virgen de Monserrate; Manta, Ecuador; diana_mero.14@hotmail.com; https://orcid.org/0000-0001-65887800

CORRESPONDENCIA

Andrea Natacha Pico Tagle

natachapicotagle@hotmail.com

Manta, Ecuador

〔C RECIMUNDO; Editorial Saberes del Conocimiento, 2021 


\section{RESUMEN}

La alteración sensorial más frecuente de las personas es la hipoacusia. Es una enfermedad con numerosas implicaciones médicas, sociales y culturales. Se denomina hipoacusia o sordera "al defecto funcional que ocurre cuando un sujeto pierde capacidad auditiva, independientemente de la intensidad. Se calcula que, a escala mundial, uno de cada 1,000 niños nace con hipoacusia bilateral profunda y cinco de cada 1,000 con otras formas de sordera. El objetivo fundamental de la presente revisión consiste en plasmar los principales síntomas de la hipoacusia en el niño, así como los factores de riesgo en el neonato. De igual manera se esboza el tratamiento del neonato y el niño diagnosticado con hipoacusia. El modelo de investigación es una revisión de tipo documental bibliográfico. E la revisión se encontró que los principales síntomas de la hipoacusia, en esta etapa de la vida, están referidos a la capacidad de percibir y emitir sonidos, de acuerdo a lo que se espera para esa edad. En el caso del neonato, en la actualidad existen protocolos de cribado que se llevan a cabo con la finalidad de detectar cualquier anormalidad lo antes posible. En cuanto a su tratamiento, múltiples literaturas han dejado en claro los beneficios de la intervención temprana. La terapéutica puede incluir audífonos o prótesis auditivas, implantes cocleares, implantes del tronco cerebral y atención temprana que incluye la intervención logopédica y educativa del niño. La hipoacusia en neonatos por su elevada incidencia y sus numerosas implicaciones se ha constituido en un importante problema de salud mundial. La pérdida o ausencia de la audición en el recién nacido conlleva a una serie de consecuencias negativas que repercutirán en el desarrollo del niño, por tanto, es fundamental la detección temprana y su intervención para garantizar la mejor calidad de vida posible a estos pacientes.

Palabras clave: Síntomas, Tratamiento, Neonato, Hipoacusia, Sordera.

\section{ABSTRACT}

The most common sensory alteration in people is hearing loss. It is a disease with numerous medical, social and cultural implications. Hearing loss or deafness is called "the functional defect that occurs when a subject loses hearing capacity, regardless of the intensity. It is estimated that, globally, one in 1,000 children is born with profound bilateral hearing loss and five in 1,000 with other forms of deafness. The main objective of this review is to capture the main symptoms of hearing loss in children, as well as the risk factors in the newborn. In the same way, the treatment of the neonate and the child diagnosed with hearing loss is outlined. The research model is a bibliographic documentary type review. In the review it was found that the main symptoms of hearing loss at this stage of life are related to the ability to perceive and make sounds, according to what is expected for that age. In the case of the newborn, there are currently screening protocols that are carried out in order to detect any abnormality as soon as possible. Regarding its treatment, multiple literatures have made clear the benefits of early intervention. Therapeutics may include hearing aids or hearing aids, cochlear implants, brainstem implants, and early care including speech therapy and education for the child. Hearing loss in neonates, due to its high incidence and its numerous implications, has become a major global health problem. The loss or absence of hearing in the newborn entails a series of negative consequences that will affect the development of the child, therefore, early detection and intervention is essential to guarantee the best possible quality of life for these patients.

Keywords: Symptoms, Treatment, Neonate, Hearing Loss, Deafness.

\section{RESUMO}

A alteração sensorial mais comum nas pessoas é a perda auditiva. Trata-se de uma doença com numerosas implicações médicas, sociais e culturais. A perda auditiva ou surdez é chamada "o defeito funcional que ocorre quando um sujeito perde a capacidade auditiva, independentemente da intensidade. Estima-se que, globalmente, uma em cada 1000 crianças nasce com perda auditiva bilateral profunda e cinco em cada 1000 com outras formas de surdez. O principal objectivo desta revisão é captar os principais sintomas da perda auditiva em crianças, bem como os factores de risco no recém-nascido. Da mesma forma, o tratamento do neonato e da criança diagnosticada com perda auditiva é delineado. O modelo de pesquisa é uma revisão de tipo documental bibliográfico. Na revisão verificou-se que os principais sintomas da perda auditiva nesta fase da vida estão relacionados com a capacidade de perceber e fazer sons, de acordo com o que se espera para essa idade. No caso do recém-nascido, existem actualmente protocolos de rastreio que são efectuados a fim de detectar qualquer anormalidade o mais rapidamente possível. Relativamente ao seu tratamento, as múltiplas literaturas deixaram claros os benefícios da intervenção precoce. A terapêutica pode incluir aparelhos auditivos ou aparelhos auditivos, implantes cocleares, implantes de tronco cerebral e cuidados precoces, incluindo terapia da fala e educação para a criança. A perda de audição em recém-nascidos, devido à sua elevada incidência e às suas numerosas implicações, tornou-se um grande problema de saúde global. A perda ou ausência de audição no recém-nascido implica uma série de consequências negativas que irão afectar o desenvolvimento da criança, pelo que a detecção precoce e a intervenção é essencial para garantir a melhor qualidade de vida possível a estes pacientes.

Palavras-chave: Sintomas, Tratamento, Neonatologia, Perda de Audição, Surdez. 


\section{Introducción}

La hipoacusia es la alteración sensorial más frecuente del ser humano. Es una enfermedad con numerosas implicaciones médicas, sociales y culturales. Para Collazo Lorduy \& Corzón Pereira, (2015) se denomina hipoacusia o sordera "al defecto funcional que ocurre cuando un sujeto pierde capacidad auditiva, independientemente de la intensidad". (p. 1)

Las deficiencias de la audición en la niñez temprana pueden causar problemas permanentes en las habilidades receptivas y expresivas del lenguaje. La gravedad de la discapacidad se determina por factores tales como:

- La edad a la que se produjo la pérdida de la audición

- Naturaleza de la pérdida (duración, frecuencias afectadas y grado)

- La susceptibilidad de cada niño en particular (p. ej., deficiencia visual coexistente, discapacidad intelectual, deficiencia primaria del lenguaje, entorno lingüístico inadecuado). (Lustig, 2019)

La mayoría de las hipoacusias o sorderas infantiles graves o profundas están presentes en el momento de nacer. Más de un $60 \%$ de estas sorderas pueden tener origen genético. Otras sorderas congénitas son debidas a situaciones que han pasado durante el embarazo o al nacer: recién nacidos con peso menor de 1500 gramos, infecciones como meningitis o infecciones durante el embarazo (rubeola, toxoplasma, citomegalovirus) o nacidos con algunas malformaciones. Estos niños tienen más riesgo de tener hipoacusia. La mayoría de los niños sordos son hijos de padres que oyen bien. (Asociación Española de Pediatría -AEP, 2020)

Más del $5 \%$ de la población mundial, es decir, 466 millones de personas en todo el mundo padecen pérdida de audición discapacitante, de las cuales 34 millones son niños. "Se estima que de aquí a 2050 más de 900 millones de personas (una de cada diez) padecerá pérdida de audición". (Organización Mundial de la Salud - OMS, 2019)

Socorro \& Contreras, (2018) estacan que, "se calcula que, a escala mundial, uno de cada 1,000 niños nace con hipoacusia bilateral profunda y cinco de cada 1,000 con otras formas de sordera". (p. 130)

A menudo es posible tratar la hipoacusia causada por problemas en el oído medio con medicamentos o cirugía. No hay cura para la hipoacusia causada por daño en el oído interno o los nervios. El pronóstico del neonato depende de la causa y gravedad de la hipoacusia. Los adelantos en audífonos y otros dispositivos, al igual que la logopedia, les permiten a muchos niños desarrollar las habilidades normales del idioma a la par que sus compañeros con audición normal. Incluso los neonatos con hipoacusia profunda pueden mejorar con la combinación correcta de tratamientos. Si el niño padece un trastorno que afecte algo más que la audición, el pronóstico depende de qué otros síntomas y problemas tenga. (Enciclopedia Médica ADAM, 2020)

"La mayor importancia de la detección oportuna se basa en los tiempos y grados en los que la plasticidad cerebral, ya que los potenciales del desarrollo lingüístico disminuyen en relación con la edad de intervención". Cuanto más tarde se inicie la intervención apropiada, más difícil será lograr un buen desarrollo del lenguaje oral que es fundamental para el desarrollo integral del individuo. (Socorro \& Contreras, 2018, p. 133)

En virtud de lo cual, resulta imperioso el conocimiento de los diferentes síntomas y factores de riesgo del neonato para detectar precozmente e intervenir la hipoacusia y prevenir las consecuencias de su falta de tratamiento en esta etapa decisiva para el individuo. 
En consecuencia, el objetivo fundamental de la presente revisión consiste en plasmar los principales síntomas de la hipoacusia en el niño, así como los factores de riesgo en el neonato. De igual manera se esboza el tratamiento del neonato y el niño diagnosticado con hipoacusia.

\section{Materiales y métodos}

Para el desarrollo del presente estudio fueron ubicados una serie de archivos digitalizados, cuya búsqueda de contenidos científicos se llevó a cabo durante el mes de enero de 2021. Dicha metodología lo ubica como un estudio de tipo documental bibliográfico.

Se formularon ecuaciones de búsqueda, con palabras clave, operadores lógicos y booleanos, en determinadas bases de datos y/o buscadores especializados, tales como: SciELO, Researchgate, entre otros. Asimismo, se utilizaron páginas con reconocimiento científico nacional e internacional en el área de la salud, tales como: Organización Mundial de la Salud (OMS), Manuales MSD, MedlinePlus, Asociación Española de Pediatría (AEP), entre otras. Algunas de las formulaciones y descriptores utilizados con los que se obtuvieron mejores resultados fueron los siguientes: "hipoacusia"; "hipoacusia en neonatos"; "síntomas de la hipoacusia en neonatos" y "tratamiento de la hipoacusia en neonatos". La información obtenida fue filtrada bajo los criterios de idioma (español); disponibilidad del contenido (completo); periodo de publicación (2011-2021), tipo de estudio: revisiones sistemáticas, de cohorte y de casos o de controles, informe de casos; y, clase de material bibliográfico (consensos, manuales, ensayos, tesis de grado, posgrado o doctorado, informes y otras clases de contenidos).

Asimismo, se seleccionaron los recursos antes mencionados en base a su producción, aval o promoción por parte de instituciones, entes, organizaciones, sociedades o asociaciones de profesionales en el área de la salud, de carácter público o privado, nacionales, internacionales o multilaterales con reconocimiento científico dentro de la comunidad de la salud.

\section{Resultados}

Signos y síntomas de la hipoacusia en niños La Asociación Española de Pediatría, (2020) refiere que, los signos de alarma en el desarrollo del lenguaje que deben hacer sospechar de la presencia de una hipoacusia son los siguientes:

- 6 meses: no emite sonidos ni balbucea

- 12 meses: no oye sonidos (timbre, teléfono)

- 15 meses: no reconoce su nombre

- 24 meses: no imita palabras simples

- 36 meses: no dice palabras y frases de dos palabras

- 48 meses: no es capaz de hacer frases sencillas.

Los signos de hipoacusia en los niños varían por edad. Es posible que un neonato con hipoacusia no se sobresalte al sonar un ruido fuerte cerca. Asimismo, puede ser que niños más grandes, que deben estar respondiendo a las voces familiares, no muestren ninguna reacción cuando se les habla. Hacia los 15 meses, los niños deben estar empleando palabras aisladas y algunas oraciones simples de 2 palabras hacia la edad de 2 años. En virtud de lo anterior, si los niños de esas edades no alcanzan estos hitos, la causa puede ser la hipoacusia. En muchos casos la hipoacusia no es diagnosticada sino hasta la edad escolar, independientemente de haber nacido o no con ella. Por último, es importante destacar que, la falta de atención y el hecho retrasarse en el trabajo con respecto al resto de la clase pueden ser signos de una hipoacusia sin diagnosticar. (Enciclopedia Médica ADAM, 2020) 
Los síntomas suelen orientar el diagnóstico de la enfermedad, no obstante, en el neonato, dada la importancia del diagnóstico y la intervención temprana, la aplicación de protocolos de cribado poblacional suele ser empleados en diferentes países, siendo obligatorio en algunos de ellos. Estos protocolos toman en consideración, entre otros, los factores de riesgo de hipoacusia del neonato para su aplicación.

Al respecto, Delgado Domínguez, (2011) manifiesta que, en los recién nacidos de riesgo (ver Tabla 1), que constituyen de un 4 a un $7 \%$ de todos los recién nacidos, se debe realizar un cribado con potenciales auditivos evocados, automatizados o no, u otoemisiones antes de los tres meses de edad corregida (teniendo en cuenta su edad gestacional). Igualmente, se estima que un $8 \%$ de los recién nacidos de alto riesgo padecerán sordera, que será de moderada-grave a profunda en el $25 \%$ de los casos. Estos criterios han sido modificados en el año 2000, agrupando varios de los definidos con anterioridad por el Joint Committee of Infant Hearing norteamericano. (p. 289)

Tabla 1. Indicadores asociados a sordera neurosensorial y/o de conducción en recién nacidos (0 a 28 días) si no se dispone de cribado universal.

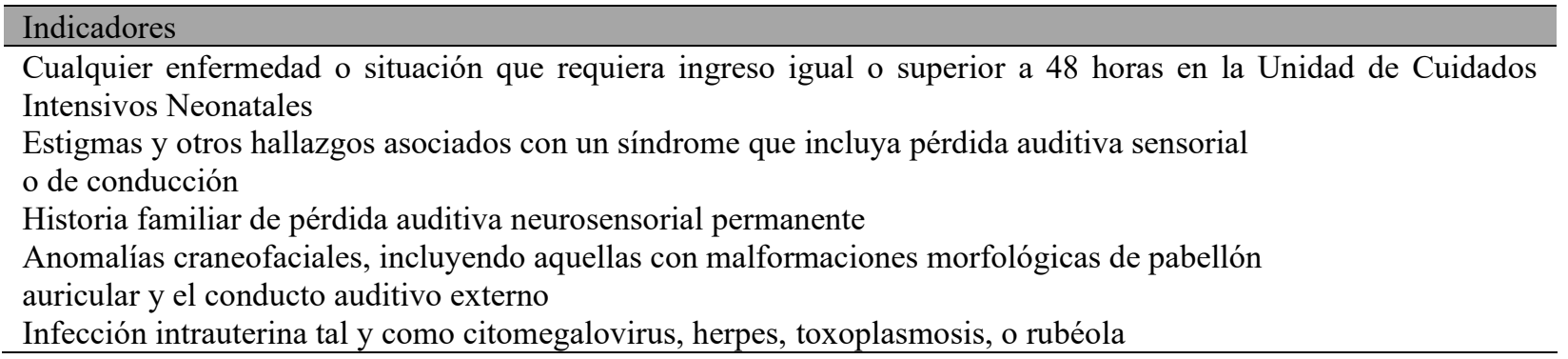

Fuente: Joint Committee of Infant Hearing norteamericano, citado por (Delgado Domínguez, 2011)

\section{Tratamiento de la hipoacusia en neonatos}

Díaz, Goycoolea, \& Cardemil, (2016) explican la importancia de la detección temprana de la hipoacusia para el tratamiento lo más tempranamente posible, sobre todo en neonatos. Asimismo, los autores refieren al respecto lo siguiente:

Hoy en día, es axiomático que la discapacidad auditiva debe ser detectada tempranamente, antes de los 3 meses de edad, con la intervención tan pronto sea posible. En el pasado, la edad media de identificación era, aproximadamente, a los 19-36 meses. Ahora, en la era del tamizaje auditivo universal, la mayoría de los estudios muestran que la edad promedio de diagnóstico ya se ha reducido de manera significativa: entre el tercer y el sexto mes de edad. Niños post era tamizaje auditivo comienza tratamiento unos 24 meses antes que los niños con deficiencia auditiva que los niños que no tuvieron tamizaje al nacer. (p. 733)

Asimismo, la OMS, (2019) al respecto agrega:

La detección e intervención tempranas son fundamentales para minimizar las consecuencias de la pérdida de audición, en el desarrollo y el rendimiento escolar del niño. En los lactantes y niños pequeños con pérdida de audición, la detección y el tratamiento tempranos en el marco de programas de detección auditiva neonatal pueden mejorar los resultados lingüísticos y escolares del niño. 
Por su parte, Baños \& Rosario, (2017) expresan que en los neonatos es fundamental tener en cuenta que el tratamiento "ha de llevarse a cabo lo antes posible, cuando la plasticidad de la vía auditiva es mayor. Iniciar el tratamiento en los primeros seis meses es especialmente crítico para lograr el máximo beneficio sobre la adquisición y desarrollo del lenguaje". (p. 25)

En general, todo el mundo está de acuerdo en que la intervención de la sordera infantil debe ser multidisciplinar. El equipo encargado de su cuidado y seguimiento (ORL, pediatra, audioprotesista, trabajador social, logopeda, maestro) mantendrá una interrelación que permita la mejor coordinación y asistencia interdisciplinar. La sordera es un problema familiar. La familia es el marco y el principal agente que impulsa el desarrollo personal, comunicativo y social del niño. El éxito de las técnicas audioprotésicas depende fundamentalmente de una correcta rehabilitación, basada en el estímulo auditivo desde la más temprana edad. (Benito \& Silva, 2017, p. 423)

\section{Prótesis auditiva}

La primera necesidad terapéutica es la adaptación de prótesis auditiva. (Benito \& Silva, 2017) explican al respecto las siguientes consideraciones:

La función de esta prótesis consistirá en ofrecer, un amplio rango de frecuencias audibles del habla a varios niveles (suave, medio e intenso) y asegurarse de que los sonidos intensos resulten confortables al usuario. El modelo más recomendable en el niño/a es el que se lleva detrás de la oreja (behind the ear-BTE-). Un audioprotesista infantil será el encargado de seleccionar y adaptar una determinada prótesis auditiva, no más tarde del mes siguiente al diagnóstico y antes de cumplir 6 meses. En los niños con hipoacusia bilateral, con independencia de su asimetría, se procurará la adaptación bilateral. La binauralidad mejora la locali- zación auditiva y la comprensión del habla. Se debe acudir a las citas del audioprotesista para evaluar el funcionamiento de la tecnología auditiva aproximadamente cada 4-6 semanas hasta obtener audiogramas estables y cada 3 meses durante los 2-3 primeros años, y determinar si el ajuste de los moldes es el adecuado, dado el rápido crecimiento del conducto auditivo externo (CAE) en los primeros años de vida. Los padres deben tener un cierto conocimiento de cómo funcionan los audífonos, disponer de recambio de pilas y comprender la importancia de llevar continuamente las prótesis para intensificar la experiencia auditiva. Se debe concienciar a las familias de que incluso las hipoacusias leves pueden tener un impacto social y educativo muy negativo. (p. 423)

\section{Implantes cocleares}

Son dispositivos electrónicos que sirven para que las personas sordas tengan una recepción de sonidos. Estos dispositivos permiten comprender los sonidos del ambiente, así como el habla de otras personas que estén a su alrededor, lo que repercute en una mejora significativa de la calidad de vida de estos pacientes. Asimismo, se puede destacar acerca de estos implantes lo siguiente:

Estos estimulan de forma directa el nervio auditivo. Con este aparato, se evita que el oído con deficiencias se atrofie aún más. Es la mejor alternativa para usar en estos casos de hipoacusias severas o profundas donde los audífonos no obtienen resultados. Este dispositivo posee dos partes: Hay una que es externa y que se coloca justo detrás de la oreja. Mientras que hay una interna que se ajusta debajo de la piel por vía quirúrgica. El aparato tiene un micrófono que capta los sonidos, un procesador que permite el habla y que selecciona y organiza los sonidos que el micrófono ha podido recibir. Asimismo, posee un transmisor y receptor, además de un estimulador, que recibe cier- 
tas señales del procesador del habla, que posteriormente se transforman en impulsos eléctricos. También hay unos electrodos que acumulan los impulsos del estimulador y que posteriormente son enviados al nervio auditivo. Los pequeños a quienes se les recomienda utilizar los implantes cocleares son aquellos que tengan graves discapacidad auditiva. El momento de la implantación lo determinará su otorrinolaringólogo especializado. Es el método más eficaz y no será necesario que se dediquen a aprender el lenguaje de señas ni mucho menos esforzarse por leer los labios de las personas. (Instituto de Otorrinolaringología y Cirugía de Cabeza y Cuello de Madrid, 2015)

\section{Implantes auditivos del tronco cerebral}

Es un dispositivo similar al implante coclear. Chóliz Ezquerro, (2015) explica los diferentes aspectos de este implante a continuación:

La placa de electrodos de este implante se sitúa sobre la pared del troncoencéfalo, en la proyección de los núcleos cocleares, para estimular directamente a estos. Esta indicado en las cofosis bilaterales por:

- Ausencia congénita del VIII para bilateral

- Sección traumática o iatrogénica del VIII par bilateral.

- Neurinomas bilaterales o para la neurofibromatosis tipo II.

- Ausencia congénita de las estructuras del oído interno.

- Fracturas del oído interno o laberintitis osificantes que impidan la colocación de los electrodos intracocleares.

Asimismo, es importante destacar que, los resultados auditivos del implante de troncoencéfalo son mucho peores que los del implante coclear, y la cirugía necesaria para su implantación tiene una morbilidad mucho mayor, lo que, unido a sus indicaciones que son mucho más escasas, hace que sea un procedimiento que se emplea en muy pocos pacientes. (p. 24)

\section{Atención temprana. Intervención logopé- dica y educativa}

Son elementos fundamentales que deben ser abordados desde la terapéutica al neonato con hipoacusia diagnosticada. Para Alzina, Aznárez, \& Huarte, (2012) estos elementos deben contemplar:

- Programas de Atención Temprana: se definen como un conjunto de actuaciones, actividades y estrategias de estimulación que favorecen el desarrollo de las potencialidades del niño, proporcionándole las experiencias que este necesita desde su nacimiento. Tienen en cuenta a la familia y al entorno del niño, facilitándoles constante información, orientación y formación sobre el desarrollo de los mismos. Estos programas se estructuran sobre la base de reas del desarrollo del niño (cognitiva, motora, lingüística, social y afectiva). En el niño con sordera, la atención prioritaria se dirige a la estimulación de las áreas auditiva y comunicativa. La audición es el medio fundamental de acceso al lenguaje oral, sin olvidar que a su vez el lenguaje contempla aspectos cognitivos, psicológicos, sociales, etc.

- Intervención logopédica: El objetivo es establecer una comunicación activa, para que el niño pueda comunicarse de forma interactiva y funcional, mediante la aplicación de estrategias de (re)habilitación comunicativa y lingüísticas adecuadas a la edad y características del niño y su familia. Representa un proceso individualizado, dentro de la evaluación e intervención multidisciplinar. Es fundamental la participación de la familia, que requiere apoyo, asesoramiento y formación. Desde el principio, es fundamental que puedan compartir con sus hijos un código comunicativo que asegure una relación rica con su entorno, tanto

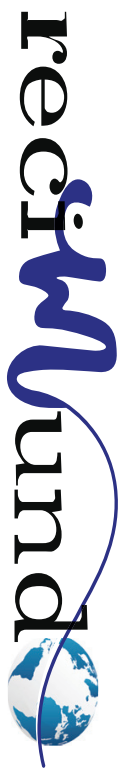


en calidad como en cantidad. Debido al avance tecnológico, en estas últimas 2 décadas de los audífonos e implantes cocleares, la intervención logopédica del niño se basa principalmente en el estímulo auditivo desde edades muy tempranas, aprovechando así el periodo de máxima plasticidad cerebral.

- La Escolarización: punto que no extenderemos en este estudio por cuanto corresponde a la edad escolar del niño. ( $p$. 341)

\section{Conclusiones}

La hipoacusia en neonatos por su elevada incidencia y sus numerosas implicaciones se ha constituido en un importante problema de salud mundial. La pérdida o ausencia de la audición en el recién nacido conlleva a una serie de consecuencias negativas que repercutirán en la calidad de vida del niño durante su crecimiento.

Estar atentos a los síntomas de la hipoacusia es importante a cualquier edad, ya que la detección temprana y su intervención le garantiza mayores probabilidades al niño de un desarrollo pleno. En cuanto a sus signos y síntomas, para esta etapa de la vida están referidos a la capacidad de percibir y emitir sonidos, de acuerdo a su edad. En el caso del neonato, en la actualidad existen protocolos de cribado que se llevan a cabo desde esta etapa con la finalidad de detectar cualquier anormalidad lo antes posible.

En cuanto a su tratamiento, múltiples literaturas han dejado en claro los beneficios de la intervención temprana. La terapéutica puede incluir audífonos o prótesis auditivas, implantes cocleares, implantes del tronco cerebral y atención temprana que incluye la intervención logopédica y educativa del niño.

\section{Bibliografía}

Alzina, V., Aznárez, N., \& Huarte, A. (2012). Tratamiento de la sordera en la infancia. Anales de Pediatría,
10(6), 334-342. Recuperado el 28 de Enero de 2021, de https://www.elsevier.es/es-revista-anales-pediatria-continuada-51-pdf-S1696281812701075

Asociación Española de Pediatría -AEP. (08 de Enero de 2020). En familia AEP. Recuperado el 10 de Enero de 2021, de https://enfamilia.aeped.es/temas-salud/sordera-hipoacusia

Baños, E., \& Rosario, M. P. (2017). Cribado neonatal de hipoacusia. Agencia de Evaluación de Tecnologías Sanitarias de Andalucía, Consejerpia de Salud, Andalucía. Recuperado el 28 de Enero de 2021, de https://www.aetsa.org/download/publicaciones/AETSA-Respuesta-Breve-Hipoacusia_DEF. pdf

Benito, J., \& Silva, J. (2017). Hipoacusia. Identificación e intervención precoz. Pediatría Integral, 21(6), 418-428. Recuperado el 29 de Enero de 2021, de https://www.pediatriaintegral.es/wp-content/uploads/2017/xxi06/03/n6-418-428_JoseBenito.pdf

Chóliz Ezquerro, M. (2015). Valoración de la eficacia de la detección precoz de la hipoacusia en la comunidad de Aragón. Tesis de grado, Universidad de Zaragoza, Facultad de Medicina de Zaragoza, Zaragoza. Recuperado el 29 de Enero de 2021, de https://core.ac.uk/download/pdf/289982352.pdf

Collazo Lorduy, T., \& Corzón Pereira, T. (2015). Evaluación del paciente con hipoacusia. En S. E. SEORL, Libro virtual de formación en otorrinolaringología SEORL (pág. 2116). Madrid, España: Sociedad Española de Otorrinolarngología y $\mathrm{Pa}$ tología Cervico Facial - SEORL PCF. Recuperado el 29 de Enero de 2021, de https://seorl.net/PDF/ Otologia/032\%20-\%20EVALUACI\%C3\%93N\%20 DEL\%2OPACIENTE\%2OCON\%2OHIPOACUSIA. pdf

Delgado Domínguez, J. J. (Junio de 2011). Detección precoz de la hipoacusia infantil. Rev Pediatr Aten Primaria , 13(50), 279-297. Recuperado el 30 de Enero de 2021, de http://scielo.isciii.es/pdf/ pap/v13n50/12_grupo_previfand.pdf

Díaz, C., Goycoolea, M., \& Cardemil, F. (2016). Hipoacusia: Incidencia, prevalencia y trascendencia. Revista Mpedica Clínica Los Condes, 27(6), 731-739. doi:https://doi.org/10.1016/j.rmclc.2016.11.003

Enciclopedia Médica ADAM. (01 de Enero de 2020). MedlinePlus. Recuperado el 08 de Enero de 2021, de https://medlineplus.gov/spanish/ency/article/007322.htm

Instituto de Otorrinolaringología y Cirugía de Cabeza y Cuello de Madrid. (2015). Instituto de Otorrino- 
laringología y Cirugía de Cabeza y Cuello de Madrid. Recuperado el 21 de Enero de 2021, de https://www.institutoorl-iom.com/hipoacusia-infantil/

Lustig, L. R. (Agosto de 2019). Manuales MSD. Recuperado el 09 de Enero de 2021, de https://www. msdmanuals.com/es/professional/trastornos-otorrinolaringol\%C3\%B3gicos/hipoacusia/hipoacusia

Organización Mundial de la Salud - OMS. (15 de Marzo de 2019). Organización Mundial de la Salud - OMS. Recuperado el 25 de Enero de 2021, de https://www.who.int/es/news-room/fact-sheets/ detail/deafness-and-hearing-loss

Socorro, A., \& Contreras, A. I. (2018). Prevalencia de hipoacusia en recién nacidos sanos en un hospital de tercer nivel de atención. Detección mediante un tamiz auditivo neonatal. Revista Mexicana de Pediatría, 85(4), 130-134. Recuperado el 15 de Enero de 2021, de https://www.medigraphic.com/ pdfs/pediat/sp-2018/sp184d.pdf

\section{CITAR ESTE ARTICULO:}

Pico Tagle, A. N., Rodríguez Centeno, J. J., Borja Zambrano, R. A., \& Mero Varela, D. A. (2021). Síntomas y tratamiento al neonato con hipoacusia. RECIMUNDO, 5(1), 313-321. https://doi.org/10.26820/recimundo/5.(1).enero.2021.313-321 